\title{
BMJ Open Quantitative systematic review of the associations between short-term exposure to nitrogen dioxide and mortality and hospital admissions
}

\author{
I C Mills, ${ }^{1}$ R W Atkinson, ${ }^{2}$ S Kang, ${ }^{2}$ H Walton, ${ }^{3,4,5}$ H R Anderson ${ }^{2,3}$
}

To cite: Mills IC,

Atkinson RW, Kang S, et al. Quantitative systematic review of the associations between short-term exposure to nitrogen dioxide and mortality and hospital admissions. BMJ Open 2015;5:e006946.

doi:10.1136/bmjopen-2014006946

- Prepublication history and additional material is available. To view please visit the journal (http://dx.doi.org/ 10.1136/bmjopen-2014006946).

Received 17 October 2014 Revised 12 February 2015 Accepted 13 February 2015

CrossMark

For numbered affiliations see end of article.

Correspondence to

IC Mills;

inga.mills@phe.gov.uk

\section{ABSTRACT}

Background: Short-term exposure to $\mathrm{NO}_{2}$ has been associated with adverse health effects and there is increasing concern that $\mathrm{NO}_{2}$ is causally related to health effects, not merely a marker of traffic-generated pollution. No comprehensive meta-analysis of the timeseries evidence on $\mathrm{NO}_{2}$ has been published since 2007.

Objective: To quantitatively assess the evidence from epidemiological time-series studies published worldwide to determine whether and to what extent short-term exposure to $\mathrm{NO}_{2}$ is associated with increased numbers of daily deaths and hospital admissions.

Design: We conducted a quantitative systematic review of 204 time-series studies of $\mathrm{NO}_{2}$ and daily mortality and hospital admissions for several diagnoses and ages, which were indexed in three bibliographic databases up to May 2011. We calculated random-effects estimates by different geographic regions and globally, and also tested for heterogeneity and small study bias.

Results: Sufficient estimates for meta-analysis were available for 43 cause-specific and age-specific combinations of mortality or hospital admissions (25 for $24 \mathrm{~h} \mathrm{NO}_{2}$ and 18 of the same combinations for $1 \mathrm{~h}$ measures). For the all-age group, a $10 \mu \mathrm{g} / \mathrm{m}^{3}$ increase in $24 \mathrm{~h} \mathrm{NO}_{2}$ was associated with increases in all-cause, cardiovascular and respiratory mortality $(0.71 \%(95 \% \mathrm{Cl} 0.43 \%$ to $1.00 \%), 0.88 \%(0.63 \%$ to $1.13 \%$ ) and $1.09 \%(0.75 \%$ to $1.42 \%)$, respectively), and with hospital admissions for respiratory $(0.57 \%$ $(0.33 \%$ to $0.82 \%))$ and cardiovascular $(0.66 \%(0.32 \%$ to $1.01 \%)$ ) diseases. Evidence of heterogeneity between geographical region-specific estimates was identified in more than half of the combinations analysed.

Conclusions: Our review provides clear evidence of health effects associated with short-term exposure to $\mathrm{NO}_{2}$ although further work is required to understand reasons for the regional heterogeneity observed. The growing literature, incorporating large multicentre studies and new evidence from less well-studied regions of the world, supports further quantitative review to assess the independence of $\mathrm{NO}_{2}$ health effects from other air pollutants.

\section{Strengths and limitations of this study}

- This is, to date, the most comprehensive, quantitative systematic review of the large time-series literature on $\mathrm{NO}_{2}$ published worldwide to provide evidence of associations between short-term exposure to $\mathrm{NO}_{2}$ and mortality and hospital admissions for a wide range of respiratory and cardiovascular diagnoses.

- It reports meta-analytical estimates both globally and for different geographical regions, as well as an assessment of heterogeneity between the region-specific estimates.

- The protocol-led approach to the identification of studies and estimates for use in meta-analysis minimised selection bias at each stage of the review.

- Meta-analysis was limited to studies which provided effect estimates in numerical, rather than graphical, form along with sufficient quantitative data to enable standardisation of estimates.

- Further work is needed to understand reasons for the regional heterogeneity observed and to quantitatively assess the independence of $\mathrm{NO}_{2}$ health effects from other air pollutants.

\section{INTRODUCTION}

Epidemiological time-series studies have found associations between daily concentrations of ambient nitrogen dioxide $\left(\mathrm{NO}_{2}\right)$ and daily mortality and morbidity outcomes. ${ }^{1-7}$ Whether these associations reflect adverse health effects of $\mathrm{NO}_{2}$ has been a matter of debate. ${ }^{1}$ The uncertainty about causality arises largely because of the close correlations between $\mathrm{NO}_{2}$ and other air pollutants in the mixture of traffic-related pollutants, some of which are more plausible toxicants. This has led to a view that $\mathrm{NO}_{2}$ may be a surrogate for fine/ultrafine particulate matter (PM) produced by motor vehicles and other combustion sources. Despite this uncertainty, recent narrative reviews of the evidence linking $\mathrm{NO}_{2}$ with health effects 
concluded that the epidemiological and toxicological evidence regarding the pollutant has sufficiently strengthened the likelihood of a causal relationship. ${ }^{1}{ }^{3}$ These reviews did not undertake any quantitative assessment of the recent time-series evidence on $\mathrm{NO}_{2}$, but instead relied on existing meta-analyses, with the last comprehensive one based on peer-reviewed papers published up to the beginning of $2006 .{ }^{6}$ Since 2006, the time-series evidence on $\mathrm{NO}_{2}$ has increased substantially, providing new data from single-city studies and from influential multicity studies from less well-studied locations, for example, Asia, ${ }^{8} 9$ and from regions previously examined, for example, Europe and Canada. ${ }^{10}{ }^{11}$ The new data on $\mathrm{NO}_{2}$ will facilitate the calculation of more robust coefficients for a range of age-specific and disease-specific end points and the examination of regional variation and heterogeneity. Such quantitative analyses for $\mathrm{NO}_{2}$ are lacking but are needed for both hazard and health impact assessments.

We conducted a systematic, quantitative review of the time-series studies of daily $\mathrm{NO}_{2}$ and daily mortality and hospital admissions indexed in bibliographic databases up to May 2011. In this paper, we focus on single pollutant model results to answer the question of whether the evidence from epidemiological time-series studies suggests adverse associations between short-term exposure to $\mathrm{NO}_{2}$ and increased numbers of daily deaths and emergency admissions to hospital. We calculated meta-analytic estimates for mortality and hospital admissions for several diagnoses and age groups, by different geographic regions, as well as globally. Whether the associations identified in this review change on adjustment for the effects of co-pollutants is a different question which requires analysis of a subset of the studies presented in this review, that is, studies reporting estimates of $\mathrm{NO}_{2}$ from single-pollutant and multipollutant models. A similar review and meta-analysis has been completed for the subset of studies that reported two-pollutant model estimates of $\mathrm{NO}_{2}$ adjusted for PM. We intend to report those findings in a separate paper.

\section{METHODS}

The full methodology and a priori protocols governing the identification of studies and effect estimates for our systematic review have been described previously, but a synopsis is provided below. ${ }^{6} 12$

\section{Identification of studies for review}

Bibliographic databases were searched to identify peerreviewed time-series papers on $\mathrm{NO}_{2}$ and daily mortality and hospital admissions indexed up to May 2011. The search criteria used are shown in the online supplementary material. Papers suitable for inclusion in the review were identified by scanning titles and abstracts to remove irrelevant citations and then reviewing full papers of remaining citations. The following inclusion criteria were applied: papers must (1) have had a minimum of 1 year of data; (2) be based on the general population; (3) have controlled for important confounding factors, including season and meteorological factors; (4) have reported regression estimates and other quantitative data for analysis.

\section{Data extraction and coding}

Data from each relevant study were entered into a Microsoft Access database (Microsoft Office 2010, Microsoft Corporation). These included details of each publication (authors, journal name, paper title, year published, etc) and data required for calculating standardised effect estimates expressed as the percentage change (and 95\% CI) in the mean number of daily events associated with a $10 \mu \mathrm{g} / \mathrm{m}^{3}$ increase in $\mathrm{NO}_{2}$ concentrations. We entered all outcomes, diagnoses (International Classification of Diseases (ICD) codes), ages and $\mathrm{NO}_{2}$ concentrations for each reported estimate. We subsequently classified all reported outcomes, diseases and ages into a smaller number of diagnostic categories and age groups to enable meta-analysis of estimates for specific outcome/disease/age combinations. ${ }^{6}$

Time-series studies often report results for several different time lags (in days) between exposure and health events and vary in the lag for the reported results for outcome/disease/age combinations. To avoid selection bias, we developed an a priori protocol for identifying the principal lag for each outcome/disease/age combination for use in our review. This was the lag highlighted by the author or stated a priori, and if this was not clear, because several lagged estimates were reported, we chose (1) the lag with the highest statistical significance, regardless of the estimate being positive or negative, or (2) the lag with the largest estimate, again, irrespective of its direction. ${ }^{6}$ If only results from cumulative or distributed lag models, that is, lags averaged over several days, were reported in a study, these were used. Further processing of data included the classification of each study into a geographical region, that is, the WHO region, in which the study was conducted (see online supplementary material).

\section{Statistical analyses}

We applied an a priori protocol for the selection of estimates for meta-analysis to avoid selection bias and duplication of studies from the same population. We gave priority to estimates from multicity studies over estimates from single-city studies and the results from a city appeared only once in a meta-analysis. The protocol also gave priority to the latest publications, the most recent study time periods, and did not take account of estimates being positive or negative (see online supplementary material). We only analysed all-year, single-pollutant model estimates.

Meta-analysis was conducted when $\geq 4$ estimates were available for an outcome/disease/age combinationincluding where a multicity estimate was available-and summary estimates were calculated using a random-effects model. ${ }^{13}$ We used a staged approach to 
Table 1 Summary of time-series studies of $\mathrm{NO}_{2}$ and mortality and hospital admissions available for review

\begin{tabular}{|c|c|c|c|c|c|c|}
\hline \multirow[b]{2}{*}{ Outcome } & \multicolumn{2}{|l|}{ Total } & \multicolumn{2}{|c|}{ Multicity study } & \multicolumn{2}{|c|}{ Single-city study } \\
\hline & Mortality* & $\begin{array}{l}\text { Hospital } \\
\text { admission }\end{array}$ & Mortality & $\begin{array}{l}\text { Hospital } \\
\text { admission }\end{array}$ & Mortality & $\begin{array}{l}\text { Hospital } \\
\text { admission }\end{array}$ \\
\hline \multicolumn{7}{|l|}{ Disease } \\
\hline Respiratory & 70 & 68 & 16 & 12 & 54 & 56 \\
\hline Cardiovascular & 84 & 52 & 18 & 14 & 66 & 38 \\
\hline \multicolumn{7}{|l|}{ WHO region } \\
\hline American A & 24 & 23 & 6 & 5 & 18 & 18 \\
\hline European A & 56 & 41 & 16 & 11 & 40 & 30 \\
\hline Western Pacific B & 31 & 17 & 2 & 2 & 29 & 15 \\
\hline American B & 7 & 5 & 0 & 0 & 7 & 5 \\
\hline Western Pacific A & 5 & 7 & 1 & 3 & 4 & 4 \\
\hline
\end{tabular}

meta-analysis, with single-city estimates pooled within WHO region prior to the pooled single-city and selected multicity estimates being pooled to produce a global estimate and WHO region-specific summary estimates. Heterogeneity between WHO region summary estimates was assessed using the $\mathrm{I}^{2}$ statistic which describes the

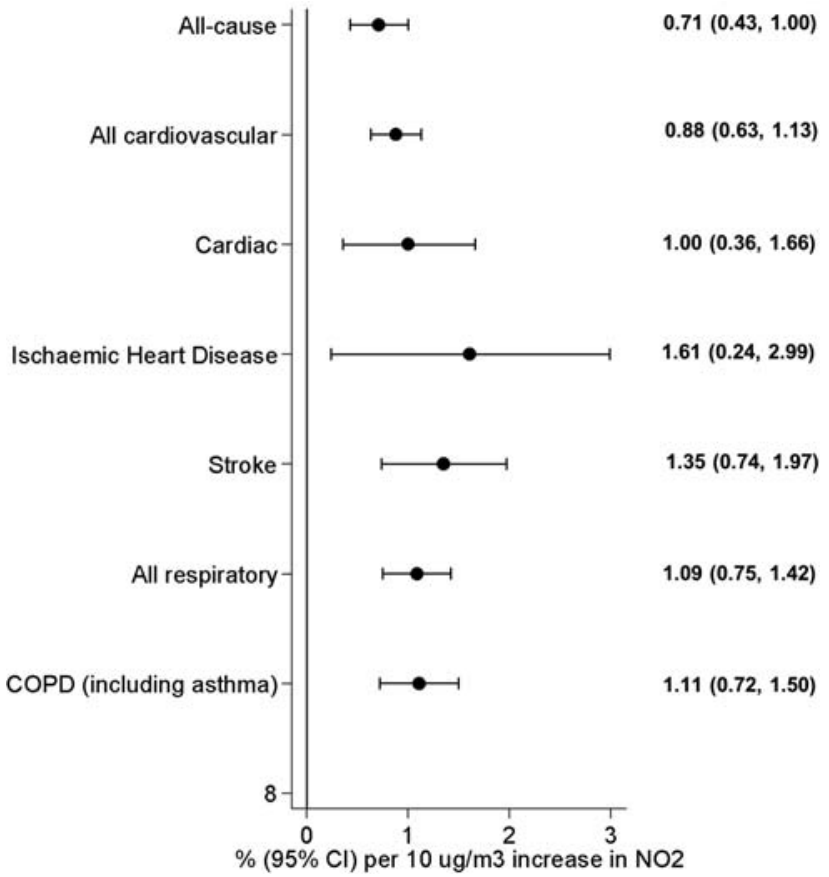

Figure 1 Random-effects summary estimates (95\% Cls) for all-cause and cause-specific mortality in all ages per $10 \mu \mathrm{g} / \mathrm{m}^{3}$ increase in $24 \mathrm{~h} \mathrm{NO}_{2}$ (COPD, chronic obstructive pulmonary disease). proportion of the variation in effect estimates attributable to between-study variation rather than chance. ${ }^{14}$ Where estimates permitted, meta-analyses were conducted for both 24 and $1 \mathrm{~h}$ average $\mathrm{NO}_{2}$. Finally, we investigated our selected single-city estimates and our pooled single-city and selected multicity estimates for evidence of small study bias using the methods of Begg and Berlin ${ }^{15}$ and Egger et al. ${ }^{16}$ We used the trim and fill technique to adjust the global random-effects estimate for small study bias where it was detected. ${ }^{17}$ This technique estimates and adjusts for the numbers and results of unreported studies. All analyses were conducted in STATA (STATA/SE V.11. StataCorp, Texas, USA).

\section{RESULTS}

Two hundred and four time-series studies of $\mathrm{NO}_{2}$ and hospital admissions and mortality indexed in bibliographic databases up to May 2011 were available for review: a list of references is provided in the online supplementary material. Table 1 summarises the numbers of studies stratified by outcome, broad disease categories, WHO region, single-city and multicity study designs, and by averaging time (24 and $1 \mathrm{~h}$ ). The majority of studies were conducted in Europe, North America and the WHO Western Pacific region and most used $24 \mathrm{~h}$ average concentrations of $\mathrm{NO}_{2}$. There were 123 and 94 studies of mortality and hospital admissions, respectively, and 42 studies used a multicity design.

\section{Mortality}

Sufficient estimates for meta-analysis were available for seven combinations of all-age, all-cause and cause-specific 
mortality and $24 \mathrm{~h} \mathrm{NO}_{2}$, four of which also provided sufficient estimates for $1 \mathrm{~h} \mathrm{NO}$ for meta-analysis.

Summary estimates for all-cause and cause-specific mortality are given in figure 1 , and for all-cause, all cardiovascular and all respiratory causes, stratified by WHO region in table 2. Estimates used in the meta-analyses for mortality are presented in forest plots in the online supplementary figures S1-S10. A $10 \mu \mathrm{g} / \mathrm{m}^{3}$ increase in $24 \mathrm{~h}$ $\mathrm{NO}_{2}$ was associated with a $0.71 \%(95 \%$ CI $0.43 \%$ to $1.00 \%)$ increase in the risk of death from all causes. This summary estimate is based on 31 single-city and multicity estimates selected for meta-analysis from the 84 identified in the review. Positive associations for all cardiovascular $(0.88 \%$ (95\% CI $0.63 \%$ to $1.13 \%)$ ) and all respiratory $(1.09 \%$ (95\% CI $0.75 \%$ to $1.42 \%)$ ) mortality were also observed, and though based on fewer estimates, we found positive summary estimates for mortality from other causes, that is, cardiac disease, ischaemic heart disease, stroke and chronic obstructive pulmonary disease (COPD) including asthma (figure 1).

Evidence of high heterogeneity in WHO regionspecific summary estimates was identified in more than half of the mortality combinations (table 2 and see online supplementary table S1). We regarded an $\mathrm{I}^{2}$ statistic of $\geq 50 \%$ as being evidence of high heterogeneity. The evidence regarding deaths from cardiac disease, ischaemic heart disease, stroke and COPD including asthma was limited to a small number of WHO regions (see online supplementary table S1). In general, larger summary estimates for mortality were found in Europe and Asia (as WHO regions Europe A, Western Pacific region and multiregion in table 2 and online supplementary table S1). Where available, summary estimates for WHO region America A, that is, USA and Canada, were consistently smaller than those from other WHO regions for all mortality combinations examined for $24 \mathrm{~h} \mathrm{NO}$.

The available evidence for $1 \mathrm{~h} \mathrm{NO}_{2}$ was smaller in comparison with that for $24 \mathrm{~h} \mathrm{NO}$. The few available estimates were mainly limited to the broad mortality combinations and, with the exception of all-cause mortality, gave comparable summary estimates (table 2).

\section{Hospital admissions}

We analysed 18 combinations of cause-specific and agespecific hospital admissions and $24 \mathrm{~h} \mathrm{NO}_{2}$, and 14 of the same combinations for $1 \mathrm{~h} \mathrm{NO}$. Figure 2 presents

Table 2 Meta-analysis results for mortality in all ages associated with a $10 \mu \mathrm{g} / \mathrm{m}^{3}$ increase in $\mathrm{NO}_{2}$

\begin{tabular}{|c|c|c|c|c|c|c|c|c|}
\hline \multirow[b]{2}{*}{ WHO region } & \multicolumn{4}{|l|}{$\mathrm{NO}_{2} 24 \mathrm{~h}$} & \multicolumn{4}{|l|}{$\mathrm{NO}_{2} 1 \mathrm{~h}$} \\
\hline & All SC/MC* & $\begin{array}{l}\text { Selected } \\
\text { SC/MC† }\end{array}$ & $\begin{array}{l}\text { Random effects } \\
(95 \% \mathrm{Cl}) \ddagger\end{array}$ & $I^{2}(\%) \S$ & All SC/MC* & $\begin{array}{l}\text { Selected } \\
\text { SC/MC }+\end{array}$ & $\begin{array}{l}\text { Random effects } \\
(95 \% \mathrm{CI}) \neq\end{array}$ & $I^{2}(\%) \S$ \\
\hline \multicolumn{9}{|c|}{ All-cause, all ages } \\
\hline Overall & $72 / 12$ & $5 / 6$ & $0.71(0.43$ to 1.00$)$ & 61.8 & $20 / 3$ & $3 / 2$ & $0.24(0.00$ to 0.49$)$ & 88.2 \\
\hline AMR A & $11 / 5$ & $1 / 2$ & $0.32(-0.01$ to 0.67$)$ & & $2 / 0$ & $2 / 0$ & $0.76(-0.05$ to 1.58$)$ & \\
\hline AMR B & $1 / 0$ & $1 / 0$ & $0.59(-0.26$ to 1.45$)$ & & $2 / 0$ & $2 / 0$ & $-0.06(-0.13,0.01)$ & \\
\hline EUR A & $40 / 6$ & $14 / 3$ & $0.90(0.45$ to 1.35$)$ & & $14 / 2$ & $3 / 1$ & $0.25(0.10$ to 0.40$)$ & \\
\hline WPR A & $3 / 0$ & $3 / 0$ & $0.66(-0.10$ to 1.44$)$ & & $2 / 1$ & $0 / 1$ & $0.58(0.21$ to 0.94$)$ & \\
\hline WPR B & $17 / 0$ & $6 / 0$ & $0.56(0.18$ to 0.94$)$ & & - & - & - & \\
\hline Multiregion ${ }^{\star \star}$ & $0 / 1$ & $0 / 1$ & $1.23(0.84$ to 1.62$)$ & & - & - & _ & \\
\hline \multicolumn{9}{|c|}{ All cardiovascular, all ages } \\
\hline Overall & $54 / 8$ & $5 / 5$ & $0.88(0.63$ to 1.13$)$ & 72.7 & $13 / 2$ & $1 / 2$ & $0.85(0.20$ to 1.50$)$ & 0 \\
\hline AMR A & $5 / 1$ & $4 / 1$ & $0.53(0.38$ to 0.67$)$ & & - & - & - & \\
\hline AMR B & $1 / 0$ & $1 / 0$ & $0.73(-0.87$ to 2.36$)$ & & - & - & - & \\
\hline EUR A & $30 / 6$ & $10 / 3$ & $1.03(0.70$ to 1.36$)$ & & $12 / 1$ & $2 / 1$ & $1.02(-0.44$ to 2.49$)$ & \\
\hline WPR A & $1 / 0$ & $1 / 0$ & $0.67(-0.24$ to 1.60$)$ & & $1 / 1$ & $0 / 1$ & $0.94(0.42$ to 1.47$)$ & \\
\hline WPR B & $17 / 0$ & $7 / 0$ & 0.91 (0.52 to 1.31$)$ & & - & - & - & \\
\hline Multiregion** & $0 / 1$ & $0 / 1$ & $1.36(0.89$ to 1.83$)$ & & - & - & - & \\
\hline \multicolumn{9}{|c|}{ All respiratory, all ages } \\
\hline Overall & $49 / 8$ & $5 / 5$ & 1.09 (0.75 to 1.42$)$ & 14.7 & $16 / 3$ & $1 / 2$ & $1.02(-0.37$ to 2.44$)$ & 87.4 \\
\hline AMR A & $4 / 1$ & $3 / 1$ & $0.54(-0.02$ to 1.10$)$ & & - & - & - & \\
\hline AMR B & $1 / 0$ & $1 / 0$ & $1.21(-1.43$ to 3.91$)$ & & - & - & - & \\
\hline EUR A & $29 / 6$ & $9 / 3$ & $1.32(0.74$ to 1.91$)$ & & $14 / 2$ & $2 / 1$ & 0.38 (0.17 to 0.59$)$ & \\
\hline WPR A & $3 / 0$ & $3 / 0$ & $1.27(-0.86$ to 3.45$)$ & & $2 / 1$ & $0 / 1$ & 2.00 (0.89 to 3.12$)$ & \\
\hline WPR B & $12 / 0$ & $5 / 0$ & 1.49 (0.47 to 2.52$)$ & & - & - & - & \\
\hline Multiregion** & $0 / 1$ & $0 / 1$ & $1.48(0.68$ to 2.29$)$ & & - & - & - & \\
\hline
\end{tabular}

${ }^{*}$ Numbers of SC/MC estimates available from all studies.

†Numbers of SC/MC estimates selected for meta-analysis.

†Random-effects summary estimate $(95 \% \mathrm{Cl})$ per $10 \mu \mathrm{g} / \mathrm{m}^{3}$ increase in $\mathrm{NO}_{2}$.

$\S I^{2}$ statistic for heterogeneity between WHO regions.

ПEstimate numbers for 'overall' refer to: (1) the number of SC/MC estimates available from all studies; (2) for selected estimates, it is the number of pooled (from SC estimates) and MC estimates used to calculate the overall summary estimate across WHO regions.

${ }^{* *}$ Multiregion refers to more than one WHO region. In this table, only one MC study (RMID 313) provided estimates for WHO multiregion for mortality; the WHO regions covered in this study were SEAR B and WPR B.

AMR, Region of the Americas; EUR, European Region; MC, multicity; SC, single-city; SEAR, South East Asian Region;

WPR, Western Pacific Region. 


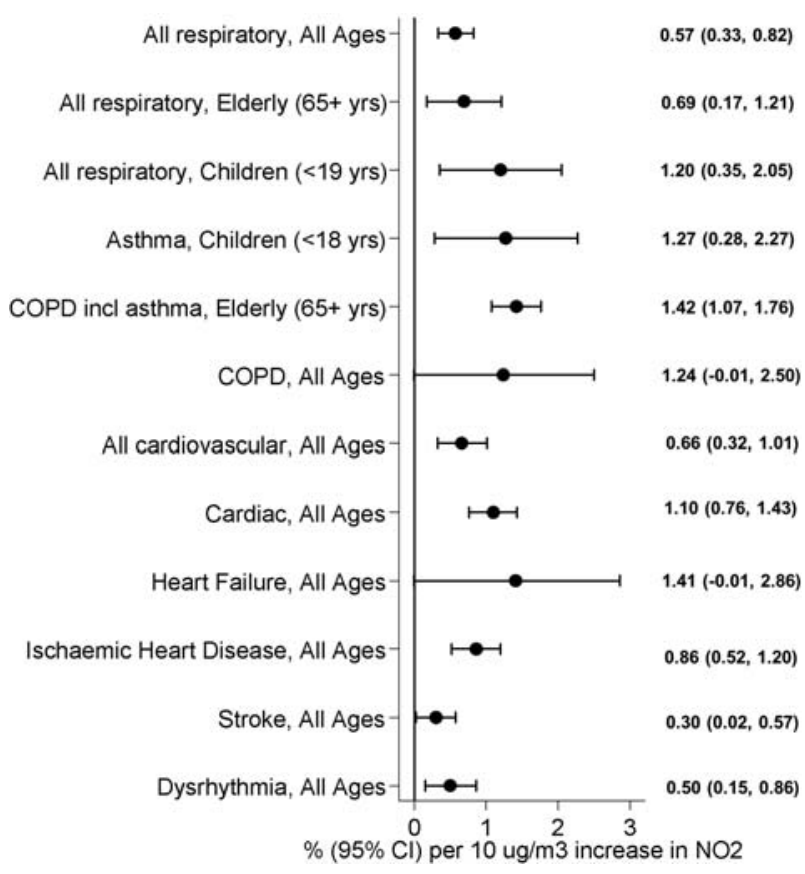

Figure 2 Random-effects summary estimates (95\% Cls) for cause-specific and age-specific hospital admissions per $10 \mu \mathrm{g} / \mathrm{m}^{3}$ increase in $24 \mathrm{~h} \mathrm{NO}_{2}$ (COPD, chronic obstructive pulmonary disease).

summary estimates for hospital admissions and $24 \mathrm{~h}$ $\mathrm{NO}_{2}$. Estimates used in the meta-analyses for hospital admissions are presented in forest plots in the online supplementary figures S11-S39.

We identified positive associations between $24 \mathrm{~h} \mathrm{NO}_{2}$ and admissions to hospital for respiratory diseases. Based on 15 estimates from five WHO regions, we found a $0.57 \%$ (95\% CI $0.33 \%$ to $0.82 \%)$ increase in hospital admissions for all respiratory diseases in all ages associated with a $10 \mu \mathrm{g} / \mathrm{m}^{3}$ increase in $24 \mathrm{~h} \mathrm{NO}$ and no evidence of heterogeneity between WHO region-specific estimates $\left(I^{2}=0 \%\right)$. Positive associations with all respiratory admissions were also observed in children and the elderly; asthma in children $(1.27 \%$ (95\% CI $0.28 \%$ to $2.27 \%)$ ); COPD including asthma in the elderly $(1.42 \%$ (95\% CI $1.07 \%$ to $1.76 \%)$ ); and for COPD in all ages $(1.24 \%(95 \%$ CI $-0.01 \%$ to $2.50 \%))$-all estimates per $10 \mu \mathrm{g} / \mathrm{m}^{3}$ increase in $24 \mathrm{~h} \mathrm{NO}$.

For all-age all cardiovascular hospital admissions, the meta-analysis of seven single-city and multicity study estimates from three WHO regions produced a summary estimate of $0.66 \%$ (95\% CI $0.32 \%$ to $1.01 \%$ ). Overall estimates for hospital admissions for specific cardiovascularrelated diseases in all ages ranged from $0.30 \%$ for stroke admissions to $1.41 \%$ for admissions for heart failure, and in all cases lower CIs were above, or very close to, $0 \%$ (figure 2). Summary estimates for all $24 \mathrm{~h}$ combinations of cardiovascular-related hospital admissions, except for stroke, in the elderly were larger than the corresponding all-age summary estimates, but there was some overlap in the CIs with those of the all-age combinations (see online supplementary table S3).
Evidence of high heterogeneity in WHO regionspecific summary estimates was identified in more than half of the 18 combinations of cause-specific and agespecific hospital admissions and $24 \mathrm{~h} \mathrm{NO}_{2}$ (see online supplementary tables S2 and S3).

The available evidence for $1 \mathrm{~h} \quad \mathrm{NO}_{2}$ and hospital admissions was smaller in comparison but showed positive associations for all combinations examined (see online supplementary tables S2 and S3).

\section{Small study bias}

Table 3 presents results of our assessment of small study bias in single-city studies within $\mathrm{WHO}$ regions and between the pooled single-city and multicity estimates for mortality. We restricted this assessment to the broad mortality combinations as sufficient estimates were available. With the exception of all respiratory mortality, there is little evidence of small study bias: adjustment for the trim and fill technique did not lead to a substantial reduction in the magnitude of the estimates, and there was no change in the summary estimate (or 95\% CI) for all cardiovascular mortality. Evidence of small study bias was found in the multicity studies of respiratory mortality despite $p$ values of 0.66 and 0.21 for the Begg and Egger tests, respectively.

\section{DISCUSSION}

This systematic review identified 204 time-series papers on $\mathrm{NO}_{2}$ and daily mortality and hospital admissions indexed in bibliographic databases up to May 2011. We undertook a total of 25 meta-analyses for $24 \mathrm{~h} \mathrm{NO}$ and combinations of all-age, all-cause and cause-specific mortality (7) and for disease-specific and age-specific hospital admissions (18). Eighteen of the 25 combinations were also analysed for $1 \mathrm{~h} \mathrm{NO}_{2}$. Positive associations between short-term exposure to $\mathrm{NO}_{2}$ and mortality from all-cause and cause-specific diseases and with cardiovascular and respiratory hospital admissions were observed. Overall, there was evidence of high heterogeneity between estimates for WHO regions in more than half of the outcomes examined.

Our quantitative assessment of the literature supports the conclusions of recent narrative reviews ${ }^{13}$ of the epidemiological evidence on $\mathrm{NO}_{2}$ and is also broadly compatible with those from the last comprehensive quantitative review by Anderson et at based on timeseries studies published up to the beginning of 2006 . The meta-analyses by Anderson et al used only single-city estimates, reflecting the dominance of this study design at the time. Our summary estimates now include a number of multicity studies, which generally applied standardised methods, conducted in different geographical regions of the world, as well as newer results from single-city studies. Our review also reflects a growing literature from outside of North America and Europe, notably from Asia, with 47 studies identified by our literature search up to May 2011. This growth is also 
Table 3 Assessment of bias in single-city studies and in pooled estimates for all-cause, all cardiovascular and all respiratory mortality, in all ages, $24 \mathrm{~h} \mathrm{NO}_{2}$

\begin{tabular}{|c|c|c|c|c|c|c|}
\hline & \multicolumn{2}{|c|}{ All-cause } & \multicolumn{2}{|c|}{ All cardiovascular } & \multicolumn{2}{|c|}{ All respiratory } \\
\hline Overall estimate no adjustment for bias* & \multicolumn{2}{|c|}{$0.71(0.43$ to 1.00$)$} & \multicolumn{2}{|c|}{$0.88(0.63$ to 1.13$)$} & \multicolumn{2}{|c|}{$1.09(0.75$ to 1.42$)$} \\
\hline \multicolumn{7}{|l|}{ Single-city bias $\dagger$} \\
\hline WHO region & EUR A & WPR B & EUR A & WPR B & EUR A & WPR B \\
\hline p Value Begg & 0.87 & 0.57 & 0.18 & 0.65 & 0.68 & 0.05 \\
\hline $\mathrm{p}$ Value Egger & 0.41 & 0.86 & 0.16 & 0.89 & 0.10 & 0.23 \\
\hline \# Estimates & 14 & 6 & 10 & 7 & 9 & 5 \\
\hline \# Trim and fill & 14 & 7 & 10 & 7 & 11 & 5 \\
\hline $\begin{array}{l}\text { Pooled single-city estimate after trim and fill } \\
\text { Multicity bias§ }\end{array}$ & \multicolumn{2}{|c|}{0.54 (0.31 to 0.77$)$} & \multicolumn{2}{|c|}{0.60 (0.47 to 0.73$)$} & \multicolumn{2}{|c|}{1.16 (0.56 to 1.77$)$} \\
\hline p Value Begg & \multicolumn{2}{|l|}{0.82} & \multicolumn{2}{|l|}{0.93} & \multicolumn{2}{|l|}{0.66} \\
\hline p Value Egger & \multicolumn{2}{|l|}{0.003} & \multicolumn{2}{|l|}{0.12} & \multicolumn{2}{|l|}{0.21} \\
\hline \# Estimates & \multicolumn{2}{|l|}{11} & \multicolumn{2}{|l|}{10} & \multicolumn{2}{|l|}{10} \\
\hline \# Trim and fill & \multicolumn{2}{|l|}{12} & \multicolumn{2}{|l|}{10} & \multicolumn{2}{|l|}{15} \\
\hline Overall estimate with adjustment for bias & \multicolumn{2}{|c|}{0.64 (0.36 to 0.92$)$} & \multicolumn{2}{|c|}{$0.88(0.63$ to 1.13$)$} & \multicolumn{2}{|c|}{$0.80(0.48$ to 1.12$)$} \\
\hline \multicolumn{7}{|c|}{$\begin{array}{l}{ }^{*} \text { Random-effects overall estimate }(95 \% \mathrm{Cl}) \text { per } 10 \mu \mathrm{g} / \mathrm{m}^{3} \mathrm{NO}_{2}(24 \mathrm{~h}) \text { without adjustment for publication bias. } \\
\dagger \text { Bias in single-city estimates within WHO region: region, Begg's test } \mathrm{p} \text { value, Egger's test } \mathrm{p} \text { value, number of estimates prior to adjustment for } \\
\text { trim and fill technique, number of estimates after adjustment for trim and fill technique. Assessment of bias in single-city estimates within } \\
\text { WHO region was not conducted in regions providing }<5 \text { estimates. } \\
\text { fOverall pooled singe-city estimate calculated after application of the trim and fill technique to the single-city estimates within WHO region. } \\
\S \text { Bias between pooled single-city estimates and multicity estimates, Begg's test } \mathrm{p} \text { value, Egger's test } \mathrm{p} \text { value, number of estimates prior to } \\
\text { adjustment for trim and fill technique, number of estimates after adjustment for trim and fill technique. } \\
\text { †Overall pooled estimate calculated after application of the trim and fill technique to single-city estimates within WHO region and between } \\
\text { pooled single-city estimates and multicity estimates. } \\
\text { EUR, European Region; WPR, Western Pacific Region. }\end{array}$} \\
\hline
\end{tabular}

reflected in meta-analyses focusing on data solely from studies of short-term exposure to air pollution including $\mathrm{NO}_{2}$ conducted in China. ${ }^{18}$ The growth in the timeseries literature since Anderson et at permitted an assessment of a broader range of outcomes in major depth, and also provides summary estimates not previously available for some outcomes, for example, cardiovascular admissions. It also enables the calculation of concentration response functions on a more substantial and broader evidence base increasing confidence in estimated concentration response functions which could be used in health impact assessment. The broader geographical representation in time-series studies also supports application of effect estimates for health impact assessment from long-term exposure cohort studies on $\mathrm{NO}_{2}$ based generally in North America and Western Europe.

We observed high heterogeneity between WHO region-specific estimates in more than half of the end points examined. We hypothesise that these geographical differences may be due to variations in population characteristics and in pollution sources, mixtures and ambient concentrations. However, additional work is required to investigate potential explanations for this heterogeneity. In the meantime, quantification of the health impact of short-term exposure to $\mathrm{NO}_{2}$ could therefore consider the use of local as well as global summary effect estimates, where a sufficient number of cities have been analysed.

Our review shows some coherence in terms of the direction, magnitude and statistical significance of the associations between mortality and hospital admissions for several diseases examined. There were however much higher estimates for stroke and respiratory mortality than hospitalisation for these diseases. In general, the interpretation of mortality versus admissions is problematic because deaths are coded to the underlying cause whereas admissions are coded to the cause of admission. In the present case, associations between $\mathrm{NO}_{2}$ and stroke mortality could be explained by effects on respiratory complications rather than on a mechanism related to stroke itself. It would require studies in the same population using associated ICD codes to further investigate this important question. Comparisons between results for mortality and admissions may also reflect systematic variation in the characteristics of the studies selected for each meta-analysis.

This review is, to date, the most comprehensive available for $\mathrm{NO}_{2}$, having worldwide coverage, examining major outcomes for several diseases and age groups, and providing meta-analytic estimates both globally and for specific geographical regions. A key strength of our review is the protocol-led approach to identifying studies and estimates for use in meta-analysis, which also sought to minimise selection bias in the different stages of the review. One feature of our approach, in common with other quantitative reviews, is the requirement that papers report numerical estimates but some fail to do so. In some instances, authors may only report statistically significant estimates. A further feature of our review is the inevitable time delay due to the necessity for extracting and processing large volumes of data from the studies. In addition, because our review has worldwide coverage and did not place any restriction on language, translation of some papers was necessary. We 
note that studies published since our literature cut-off date continue to provide evidence of positive associations between $\mathrm{NO}_{2}$ and daily mortality and hospital admissions. Recent multicity studies of $\mathrm{NO}_{2}$ and allcause mortality reported increases (per $10 \mu \mathrm{g} / \mathrm{m}^{3} \mathrm{NO}_{2}$ ) of $2.09 \%(95 \%$ CI $0.96 \%$ to $3.24 \%)$ in 10 cities in Italy; ${ }^{19} 1.10 \%$ (95\% CI $0.63 \%$ to $1.58 \%$ ) in 25 cities in Italy; ${ }^{20} 1.63 \%$ (95\% posterior interval $1.09 \%$ to $2.17 \%$ ) in 17 Chinese cities $^{21}$ and $1.03 \%$ ( $(95 \%$ CI $0.91 \%$ to $1.18 \%$ ) per $10 \mathrm{ppb} \mathrm{NO}_{2}$ ) in $72 \mathrm{US}$ cities. ${ }^{22}$ Some of the cities investigated in these newer multicity studies had already contributed estimates to the present meta-analysis.

The causality of adverse health associations of $\mathrm{NO}_{2}$ is a matter of current debate since there are close correlations between $\mathrm{NO}_{2}$ and other air pollutants in the mixture of traffic-related pollutants, especially PM. ${ }^{1}$ The extent of these correlations varies from city-to-city and over time, due to variations in the emission sources (eg, dieselisation of cars and primary production of $\mathrm{NO}_{2}$ by certain particle traps). Multipollutant models have been used to attempt to identify effects of $\mathrm{NO}_{2}$ that may be independent of PM and other co-pollutants. A previous meta-analysis of multipollutant model estimates of $\mathrm{NO}_{2}$ indicated a reduction in the pooled estimate and loss of statistical significance following adjustment for co-pollutants. ${ }^{23}{ }^{24}$ However, recent qualitative assessments suggest that the evidence for independent effects of $\mathrm{NO}_{2}$ is strengthening, but no recent quantitative analysis of multipollutant model estimates of $\mathrm{NO}_{2}$ has been undertaken. ${ }^{1} 3625$ The current meta-analysis concentrated on results from single-pollutant models, since these provided the greatest number of cities, but we have completed a similar systematic review and meta-analysis of the subset of studies that reported twopollutant model estimates of $\mathrm{NO}_{2}$ adjusted for PM. The purpose of this second review was to assess the extent to which the associations identified in this review change on adjustment for the effects of PM. We intend to report those findings in a separate paper.

Our review has established that there are positive and statistically significant associations between short-term exposure to $\mathrm{NO}_{2}$ and mortality and hospital admissions for a range of respiratory and cardiovascular diseases in different age groups. This comprehensive quantitative assessment of the time-series evidence on $\mathrm{NO}_{2}$ forms an important part of understanding whether $\mathrm{NO}_{2}$ contributes to the public health burden of air pollution. Further work is needed to understand reasons for the regional heterogeneity observed and to quantitatively assess the independence of $\mathrm{NO}_{2}$ health effects from other air pollutants.

\section{Author affiliations}

${ }^{1}$ Public Health England, Centre for Radiation, Chemical and Environmental Hazards, Oxfordshire, UK

${ }^{2}$ Population Health Research Institute and MRC-PHE Centre for Environment and Health, St George's, University of London, London, UK
${ }^{3}$ MRC-PHE Centre for Environment and Health, King's College London, London, UK

${ }^{4}$ NIHR Biomedical Research Centre at Guy's and St Thomas' NHS Foundation Trust and King's College London, London, UK

${ }^{5}$ NIHR Health Protection Research Unit on Environmental Hazards at King's College London in partnership with Public Health England, London, UK

Acknowledgements This is independent research commissioned by the Policy Research Programme (PRP) in the Department of Health. The views expressed are not necessarily those of the Department

Contributors All authors contributed to the design of the study, to the drafting of the paper and have seen and approved the final version. ICM read all papers, checked data prior to meta-analysis and carried out all analyses. RWA produced the statistical code in STATA used by ICM in the analyses. ICM is responsible for the overall content as lead author of the paper.

Funding Policy Research Programme (PRP) in the Department of Health (grant number PRP 002/037).

Competing interests None declared.

Provenance and peer review Not commissioned; externally peer reviewed.

Data sharing statement No additional data are available.

Open Access This is an Open Access article distributed in accordance with the Creative Commons Attribution Non Commercial (CC BY-NC 4.0) license, which permits others to distribute, remix, adapt, build upon this work noncommercially, and license their derivative works on different terms, provided the original work is properly cited and the use is non-commercial. See: http:// creativecommons.org/licenses/by-nc/4.0/

\section{REFERENCES}

1. World Health Organization (WHO) Regional Office for Europe. Review of Evidence on Health Aspects of Air Pollution-REVIHAAP Project: final technical report. 2013. http://www.euro.who.int/en/ what-we-do/health-topics/environment-and-health/air-quality/ publications/2013/review-of-evidence-on-health-aspects-of-airpollution-revihaap-project-final-technical-report (accessed Aug 2014).

2. World Health Organization (WHO) Regional Office for Europe. Air Quality Guidelines Global Update 2005: particulate matter, ozone, nitrogen dioxide and sulfur dioxide. Copenhagen: WHO Regional Office for Europe, 2006. http://www.euro.who.int/_data/assets/pdf_ file/0005/78638/E90038.pdf (accessed Aug 2014).

3. U.S. EPA. Integrated Science Assessment for Oxides of NitrogenHealth Criteria (first external review draft). Washington, DC: U.S. Environmental Protection Agency, EPA/600/R-13/202, 2013. http:// epa.gov/ncea/isa/index.htm (accessed Aug 2014).

4. U.S. EPA. Integrated Science Assessment for Oxides of NitrogenHealth Criteria (final report). Washington, DC: U.S. Environmental Protection Agency, EPA/600/R-08/071, 2008. http://cfpub.epa.gov/ ncea/isa/recordisplay.cfm?deid=194645 (accessed Jun 2014).

5. HEI International Scientific Oversight Committee. Outdoor air pollution and health in the developing countries of Asia: a comprehensive review. Special Report 18. Boston, MA: Health Effects Institute, 2010.

6. Anderson HR, Atkinson RW, Bremner SA, et al. Quantitative systematic review of short-term associations between ambient air pollution (particulate matter, ozone, nitrogen dioxide, sulphur dioxide and carbon monoxide), and mortality and morbidity. London: Department of Health, 2007. https://www.gov.uk/government/ publications/quantitative-systematic-review-of-short-termassociations-between-ambient-air-pollution-particulate-matter-ozonenitrogen-dioxide-sulphur-dioxide-and-carbon-monoxide-andmortality-and-morbidity (accessed Aug 2014).

7. California Environmental Protection Agency. Review of the California ambient air quality standard for nitrogen dioxide. Sacramento: California Environmental Protection Agency Air Resources Board, 2007. http://www.arb.ca.gov/research/aaqs/no2-rs/no2-doc. htm\#TechSuppDoc (accessed Aug 2014).

8. HEI Public Health and Air Pollution in Asia Program. Public Health and Air Pollution in Asia (PAPA): coordinated studies of short-term exposure to air pollution and daily mortality in four cities. HEI Research Report 154. Boston, MA: Health Effects Institute, 2010. 
9. Wong $\mathrm{CM}$, Vichit-Vadakan $\mathrm{N}$, Kan $\mathrm{H}$, et al. Public health and air pollution in Asia (PAPA): a multicity study of short-term effects of air pollution on mortality. Environ Health Perspect 2008;116:1195-202.

10. Samoli E, Aga E, Touloumi G, et al. Short-term effects of nitrogen dioxide on mortality: an analysis within the APHEA project. Eur Respir J 2006;27:1129-38.

11. Brook JR, Burnett RT, Dann TF, et al. Further interpretation of the acute effect of nitrogen dioxide observed in Canadian time-series studies. J Expo Sci Environ Epidemiol 2007;17(Suppl 2):S36-44.

12. Atkinson RW, Kang S, Anderson HR, et al. Epidemiological time series studies of $\mathrm{PM}_{2.5}$ and daily mortality and hospital admissions: a systematic review and meta-analysis. Thorax 2014;69:660-5.

13. DerSimonian R, Laird N. Meta-analysis in clinical trials. Control Clin Trials 1986;7:177-88.

14. Huedo-Medina TB, Sanchez-Meca J, Marın-Martınez F, et al. Assessing heterogeneity in meta-analysis: $Q$ statistic or $l^{2}$ index? Psychol Methods 2006;11:193-206.

15. Begg CB, Berlin JA. Publication bias and dissemination of clinical research. J Natl Cancer Inst 1989;81:107-15.

16. Egger M, Davey Smith G, Schneider M, et al. Bias in meta-analysis detected by a simple, graphical test. BMJ 1997:315:629-34.

17. Duval S, Tweedie R. A nonparametric "trim and fill" method of accounting for publication bias in meta-analysis. J Am Stat Assoc 2000;95:89-98.

18. Shang Y, Sun Z, Cao J, et al. Systematic review of Chinese studies of short-term exposure to air pollution and daily mortality. Environ In 2013;54:100-11.
19. Chiusolo M, Cadum E, Stafoggia M, et al; EpiAir Collaborative Group. Short-term effects of nitrogen dioxide on mortality and susceptibility factors in 10 Italian cities: the EpiAir study. Environ Health Perspect 2011;119:1233-8.

20. Alessandrini ER, Faustini $A$, Chiusolo $M$, et al. Air pollution and mortality in twenty-five Italian cities: results of the EpiAir2 project. Epidemiol Prev 2013;37:220-9.

21. Chen R, Samoli E, Wong CM, et al; CAPES Collaborative Group. Associations between short-term exposure to nitrogen dioxide and mortality in 17 Chinese cities: the China Air Pollution and Health Effects Study (CAPES). Environ Int 2012;45:32-8.

22. Moolgavkar SH, McClellan RO, Dewanji A, et al Time-series analyses of air pollution and mortality in the United States: a subsampling approach. Environ Health Perspect 2013;121:73-8

23. Stieb DM, Judek S, Burnett RT. Meta-analysis of time-series studies of air pollution and mortality: effects of gases and particles and the influence of cause of death, age, and season. J Air Waste Manag Assoc 2002;52:470-84.

24. Stieb DM, Judek S, Burnett RT. Meta-analysis of time-series studies of air pollution and mortality: update in relation to the use of generalized additive models. $J$ Air Waste Manag Assoc 2003;53:258-61.

25. Committee on the Medical Effects of Air Pollutants (COMEAP). Cardiovascular disease and air pollution. 2006. http://www.comeap. org.uk/documents (accessed 18 Mar 2013). 


\section{Correction}

Mills IC, Atkinson RW, Kang S, et al. Quantitative systematic review of the associations between short-term exposure to nitrogen dioxide and mortality and hospital admissions. BMJ Open 2015;5:e006946. There is an error in figure 1 of this article. All of the estimates and figure title are correct. The corrected figure is below.

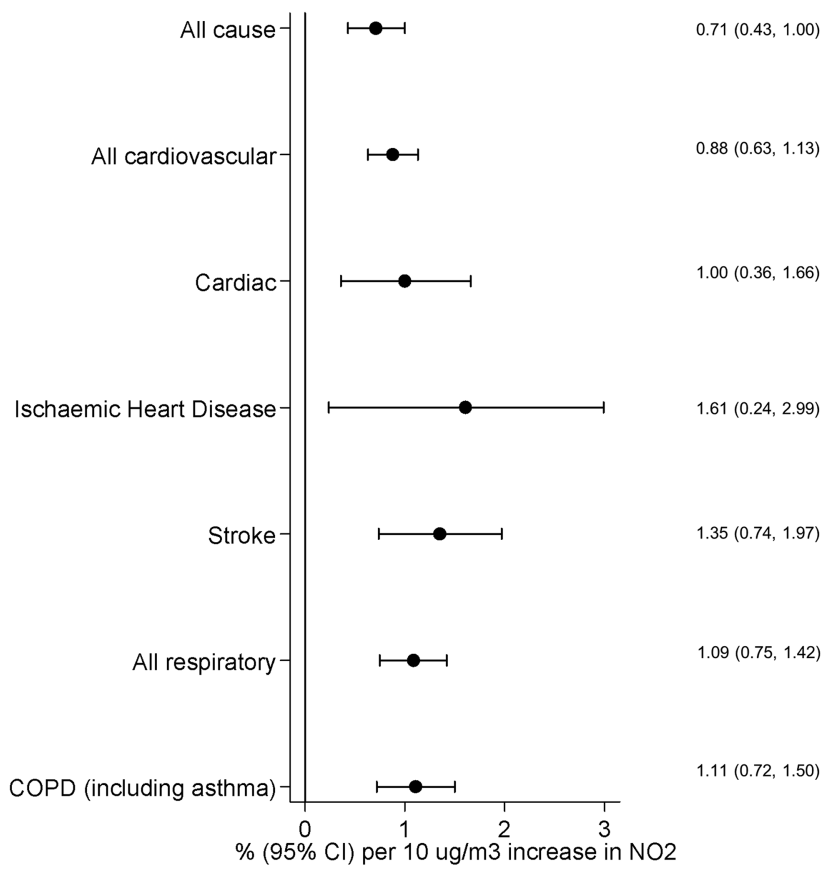

Figure 1

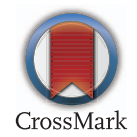

BMJ Open 2015;5:e006946. doi:10.1136/bmjopen-2014-006946corr1 